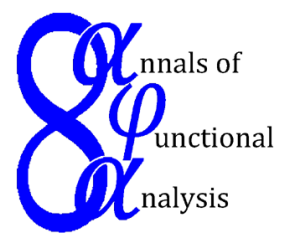

Ann. Funct. Anal. 7 (2016), no. 1, 158-169

http://dx.doi.org/10.1215/20088752-3429526

ISSN: 2008-8752 (electronic)

http://projecteuclid.org/afa

\title{
REALIZATION OF COMPACT SPACES AS CB-HELSON SETS
}

\author{
YEMON CHOI \\ Dedicated to Professor Anthony To-Ming Lau, with thanks for all his work on behalf of the \\ international community in abstract harmonic analysis \\ Communicated by Z. Lykova
}

\begin{abstract}
We show that, given a compact Hausdorff space $\Omega$, there is a compact group $\mathbb{G}$ and a homeomorphic embedding of $\Omega$ into $\mathbb{G}$, such that the restriction map $\mathrm{A}(\mathbb{G}) \rightarrow C(\Omega)$ is a complete quotient map of operator spaces. In particular, this shows that there exist compact groups which contain infinite cb-Helson subsets, answering a question raised by Choi and Samei. A negative result from that paper is also improved.
\end{abstract}

\section{INTRODUCTION}

The notion of a cb-Helson subset of a locally compact group was introduced in [4], in connection with the study of quotients of Fourier algebras. For instance, the following result can be obtained by an easy modification of the proof of $[4$, Theorem B].

Theorem 1.1 (Corollary of work in [4]). Let $G$ be a SIN group, and let $J$ be a closed ideal in the Fourier algebra $\mathrm{A}(G)$. Suppose that $\mathrm{A}(G) / J$ is completely boundedly isomorphic to a closed subalgebra of $\mathcal{B}(\mathcal{H})$ for some Hilbert space $\mathcal{H}$. Then there is a cb-Helson subset $E \subset G$ such that $J=\left\{f \in \mathrm{A}(G):\left.f\right|_{E}=0\right\}$.

We defer the definition of a cb-Helson set to Section 2. For now, we note that such sets appear to be rather hard to come by: finite subsets of locally compact groups have the cb-Helson property, but hitherto no infinite cb-Helson sets were

Copyright 2016 by the Tusi Mathematical Research Group.

Received Apr. 9, 2015; Accepted Jul. 30, 2015.

2010 Mathematics Subject Classification. Primary 43A30; Secondary 46L07.

Keywords. Fourier algebra, Helson set, operator space. 


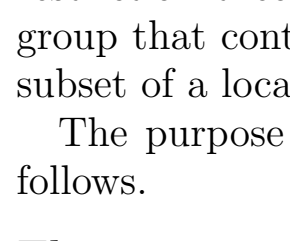

Ann. Funct. Anal. 7 (2016), no. 1, 158-169

http://dx.doi.org/10.1215/20088752-3429526

ISSN: 2008-8752 (electronic)

http://projecteuclid.org/afa

\title{
REALIZATION OF COMPACT SPACES AS CB-HELSON SETS
}

\author{
YEMON CHOI \\ Dedicated to Professor Anthony To-Ming Lau, with thanks for all his work on behalf of the \\ international community in abstract harmonic analysis \\ Communicated by Z. Lykova
}

\begin{abstract}
We show that, given a compact Hausdorff space $\Omega$, there is a compact group $\mathbb{G}$ and a homeomorphic embedding of $\Omega$ into $\mathbb{G}$, such that the restriction map $\mathrm{A}(\mathbb{G}) \rightarrow C(\Omega)$ is a complete quotient map of operator spaces. In particular, this shows that there exist compact groups which contain infinite cb-Helson subsets, answering a question raised by Choi and Samei. A negative result from that paper is also improved.
\end{abstract}

\section{INTRODUCTION}

The notion of a cb-Helson subset of a locally compact group was introduced in [4], in connection with the study of quotients of Fourier algebras. For instance, the following result can be obtained by an easy modification of the proof of $[4$, Theorem B].

Theorem 1.1 (Corollary of work in [4]). Let $G$ be a SIN group, and let $J$ be a closed ideal in the Fourier algebra $\mathrm{A}(G)$. Suppose that $\mathrm{A}(G) / J$ is completely boundedly isomorphic to a closed subalgebra of $\mathcal{B}(\mathcal{H})$ for some Hilbert space $\mathcal{H}$. Then there is a cb-Helson subset $E \subset G$ such that $J=\left\{f \in \mathrm{A}(G):\left.f\right|_{E}=0\right\}$.

We defer the definition of a cb-Helson set to Section 2. For now, we note that such sets appear to be rather hard to come by: finite subsets of locally compact groups have the cb-Helson property, but hitherto no infinite cb-Helson sets were

Copyright 2016 by the Tusi Mathematical Research Group.

Received Apr. 9, 2015; Accepted Jul. 30, 2015.

2010 Mathematics Subject Classification. Primary 43A30; Secondary 46L07.

Keywords. Fourier algebra, Helson set, operator space. 


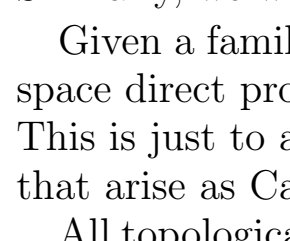

Ann. Funct. Anal. 7 (2016), no. 1, 158-169

http://dx.doi.org/10.1215/20088752-3429526

ISSN: 2008-8752 (electronic)

http://projecteuclid.org/afa

\title{
REALIZATION OF COMPACT SPACES AS CB-HELSON SETS
}

\author{
YEMON CHOI \\ Dedicated to Professor Anthony To-Ming Lau, with thanks for all his work on behalf of the \\ international community in abstract harmonic analysis \\ Communicated by Z. Lykova
}

\begin{abstract}
We show that, given a compact Hausdorff space $\Omega$, there is a compact group $\mathbb{G}$ and a homeomorphic embedding of $\Omega$ into $\mathbb{G}$, such that the restriction map $\mathrm{A}(\mathbb{G}) \rightarrow C(\Omega)$ is a complete quotient map of operator spaces. In particular, this shows that there exist compact groups which contain infinite cb-Helson subsets, answering a question raised by Choi and Samei. A negative result from that paper is also improved.
\end{abstract}

\section{INTRODUCTION}

The notion of a cb-Helson subset of a locally compact group was introduced in [4], in connection with the study of quotients of Fourier algebras. For instance, the following result can be obtained by an easy modification of the proof of $[4$, Theorem B].

Theorem 1.1 (Corollary of work in [4]). Let $G$ be a SIN group, and let $J$ be a closed ideal in the Fourier algebra $\mathrm{A}(G)$. Suppose that $\mathrm{A}(G) / J$ is completely boundedly isomorphic to a closed subalgebra of $\mathcal{B}(\mathcal{H})$ for some Hilbert space $\mathcal{H}$. Then there is a cb-Helson subset $E \subset G$ such that $J=\left\{f \in \mathrm{A}(G):\left.f\right|_{E}=0\right\}$.

We defer the definition of a cb-Helson set to Section 2. For now, we note that such sets appear to be rather hard to come by: finite subsets of locally compact groups have the cb-Helson property, but hitherto no infinite cb-Helson sets were

Copyright 2016 by the Tusi Mathematical Research Group.

Received Apr. 9, 2015; Accepted Jul. 30, 2015.

2010 Mathematics Subject Classification. Primary 43A30; Secondary 46L07.

Keywords. Fourier algebra, Helson set, operator space. 


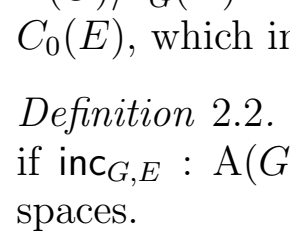

Ann. Funct. Anal. 7 (2016), no. 1, 158-169

http://dx.doi.org/10.1215/20088752-3429526

ISSN: 2008-8752 (electronic)

http://projecteuclid.org/afa

\title{
REALIZATION OF COMPACT SPACES AS CB-HELSON SETS
}

\author{
YEMON CHOI \\ Dedicated to Professor Anthony To-Ming Lau, with thanks for all his work on behalf of the \\ international community in abstract harmonic analysis \\ Communicated by Z. Lykova
}

\begin{abstract}
We show that, given a compact Hausdorff space $\Omega$, there is a compact group $\mathbb{G}$ and a homeomorphic embedding of $\Omega$ into $\mathbb{G}$, such that the restriction map $\mathrm{A}(\mathbb{G}) \rightarrow C(\Omega)$ is a complete quotient map of operator spaces. In particular, this shows that there exist compact groups which contain infinite cb-Helson subsets, answering a question raised by Choi and Samei. A negative result from that paper is also improved.
\end{abstract}

\section{INTRODUCTION}

The notion of a cb-Helson subset of a locally compact group was introduced in [4], in connection with the study of quotients of Fourier algebras. For instance, the following result can be obtained by an easy modification of the proof of $[4$, Theorem B].

Theorem 1.1 (Corollary of work in [4]). Let $G$ be a SIN group, and let $J$ be a closed ideal in the Fourier algebra $\mathrm{A}(G)$. Suppose that $\mathrm{A}(G) / J$ is completely boundedly isomorphic to a closed subalgebra of $\mathcal{B}(\mathcal{H})$ for some Hilbert space $\mathcal{H}$. Then there is a cb-Helson subset $E \subset G$ such that $J=\left\{f \in \mathrm{A}(G):\left.f\right|_{E}=0\right\}$.

We defer the definition of a cb-Helson set to Section 2. For now, we note that such sets appear to be rather hard to come by: finite subsets of locally compact groups have the cb-Helson property, but hitherto no infinite cb-Helson sets were

Copyright 2016 by the Tusi Mathematical Research Group.

Received Apr. 9, 2015; Accepted Jul. 30, 2015.

2010 Mathematics Subject Classification. Primary 43A30; Secondary 46L07.

Keywords. Fourier algebra, Helson set, operator space. 


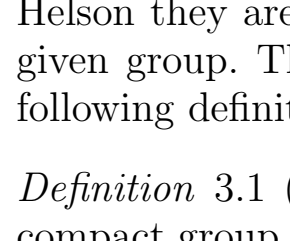

Ann. Funct. Anal. 7 (2016), no. 1, 158-169

http://dx.doi.org/10.1215/20088752-3429526

ISSN: 2008-8752 (electronic)

http://projecteuclid.org/afa

\title{
REALIZATION OF COMPACT SPACES AS CB-HELSON SETS
}

\author{
YEMON CHOI \\ Dedicated to Professor Anthony To-Ming Lau, with thanks for all his work on behalf of the \\ international community in abstract harmonic analysis \\ Communicated by Z. Lykova
}

\begin{abstract}
We show that, given a compact Hausdorff space $\Omega$, there is a compact group $\mathbb{G}$ and a homeomorphic embedding of $\Omega$ into $\mathbb{G}$, such that the restriction map $\mathrm{A}(\mathbb{G}) \rightarrow C(\Omega)$ is a complete quotient map of operator spaces. In particular, this shows that there exist compact groups which contain infinite cb-Helson subsets, answering a question raised by Choi and Samei. A negative result from that paper is also improved.
\end{abstract}

\section{INTRODUCTION}

The notion of a cb-Helson subset of a locally compact group was introduced in [4], in connection with the study of quotients of Fourier algebras. For instance, the following result can be obtained by an easy modification of the proof of $[4$, Theorem B].

Theorem 1.1 (Corollary of work in [4]). Let $G$ be a SIN group, and let $J$ be a closed ideal in the Fourier algebra $\mathrm{A}(G)$. Suppose that $\mathrm{A}(G) / J$ is completely boundedly isomorphic to a closed subalgebra of $\mathcal{B}(\mathcal{H})$ for some Hilbert space $\mathcal{H}$. Then there is a cb-Helson subset $E \subset G$ such that $J=\left\{f \in \mathrm{A}(G):\left.f\right|_{E}=0\right\}$.

We defer the definition of a cb-Helson set to Section 2. For now, we note that such sets appear to be rather hard to come by: finite subsets of locally compact groups have the cb-Helson property, but hitherto no infinite cb-Helson sets were

Copyright 2016 by the Tusi Mathematical Research Group.

Received Apr. 9, 2015; Accepted Jul. 30, 2015.

2010 Mathematics Subject Classification. Primary 43A30; Secondary 46L07.

Keywords. Fourier algebra, Helson set, operator space. 


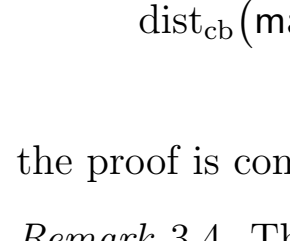

Ann. Funct. Anal. 7 (2016), no. 1, 158-169

http://dx.doi.org/10.1215/20088752-3429526

ISSN: 2008-8752 (electronic)

http://projecteuclid.org/afa

\title{
REALIZATION OF COMPACT SPACES AS CB-HELSON SETS
}

\author{
YEMON CHOI \\ Dedicated to Professor Anthony To-Ming Lau, with thanks for all his work on behalf of the \\ international community in abstract harmonic analysis \\ Communicated by Z. Lykova
}

\begin{abstract}
We show that, given a compact Hausdorff space $\Omega$, there is a compact group $\mathbb{G}$ and a homeomorphic embedding of $\Omega$ into $\mathbb{G}$, such that the restriction map $\mathrm{A}(\mathbb{G}) \rightarrow C(\Omega)$ is a complete quotient map of operator spaces. In particular, this shows that there exist compact groups which contain infinite cb-Helson subsets, answering a question raised by Choi and Samei. A negative result from that paper is also improved.
\end{abstract}

\section{INTRODUCTION}

The notion of a cb-Helson subset of a locally compact group was introduced in [4], in connection with the study of quotients of Fourier algebras. For instance, the following result can be obtained by an easy modification of the proof of $[4$, Theorem B].

Theorem 1.1 (Corollary of work in [4]). Let $G$ be a SIN group, and let $J$ be a closed ideal in the Fourier algebra $\mathrm{A}(G)$. Suppose that $\mathrm{A}(G) / J$ is completely boundedly isomorphic to a closed subalgebra of $\mathcal{B}(\mathcal{H})$ for some Hilbert space $\mathcal{H}$. Then there is a cb-Helson subset $E \subset G$ such that $J=\left\{f \in \mathrm{A}(G):\left.f\right|_{E}=0\right\}$.

We defer the definition of a cb-Helson set to Section 2. For now, we note that such sets appear to be rather hard to come by: finite subsets of locally compact groups have the cb-Helson property, but hitherto no infinite cb-Helson sets were

Copyright 2016 by the Tusi Mathematical Research Group.

Received Apr. 9, 2015; Accepted Jul. 30, 2015.

2010 Mathematics Subject Classification. Primary 43A30; Secondary 46L07.

Keywords. Fourier algebra, Helson set, operator space. 


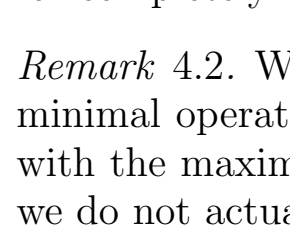

Ann. Funct. Anal. 7 (2016), no. 1, 158-169

http://dx.doi.org/10.1215/20088752-3429526

ISSN: 2008-8752 (electronic)

http://projecteuclid.org/afa

\title{
REALIZATION OF COMPACT SPACES AS CB-HELSON SETS
}

\author{
YEMON CHOI \\ Dedicated to Professor Anthony To-Ming Lau, with thanks for all his work on behalf of the \\ international community in abstract harmonic analysis \\ Communicated by Z. Lykova
}

\begin{abstract}
We show that, given a compact Hausdorff space $\Omega$, there is a compact group $\mathbb{G}$ and a homeomorphic embedding of $\Omega$ into $\mathbb{G}$, such that the restriction map $\mathrm{A}(\mathbb{G}) \rightarrow C(\Omega)$ is a complete quotient map of operator spaces. In particular, this shows that there exist compact groups which contain infinite cb-Helson subsets, answering a question raised by Choi and Samei. A negative result from that paper is also improved.
\end{abstract}

\section{INTRODUCTION}

The notion of a cb-Helson subset of a locally compact group was introduced in [4], in connection with the study of quotients of Fourier algebras. For instance, the following result can be obtained by an easy modification of the proof of $[4$, Theorem B].

Theorem 1.1 (Corollary of work in [4]). Let $G$ be a SIN group, and let $J$ be a closed ideal in the Fourier algebra $\mathrm{A}(G)$. Suppose that $\mathrm{A}(G) / J$ is completely boundedly isomorphic to a closed subalgebra of $\mathcal{B}(\mathcal{H})$ for some Hilbert space $\mathcal{H}$. Then there is a cb-Helson subset $E \subset G$ such that $J=\left\{f \in \mathrm{A}(G):\left.f\right|_{E}=0\right\}$.

We defer the definition of a cb-Helson set to Section 2. For now, we note that such sets appear to be rather hard to come by: finite subsets of locally compact groups have the cb-Helson property, but hitherto no infinite cb-Helson sets were

Copyright 2016 by the Tusi Mathematical Research Group.

Received Apr. 9, 2015; Accepted Jul. 30, 2015.

2010 Mathematics Subject Classification. Primary 43A30; Secondary 46L07.

Keywords. Fourier algebra, Helson set, operator space. 


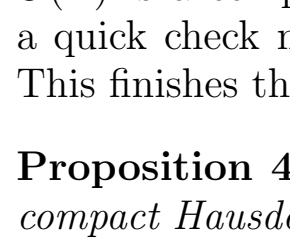

Ann. Funct. Anal. 7 (2016), no. 1, 158-169

http://dx.doi.org/10.1215/20088752-3429526

ISSN: 2008-8752 (electronic)

http://projecteuclid.org/afa

\title{
REALIZATION OF COMPACT SPACES AS CB-HELSON SETS
}

\author{
YEMON CHOI \\ Dedicated to Professor Anthony To-Ming Lau, with thanks for all his work on behalf of the \\ international community in abstract harmonic analysis \\ Communicated by Z. Lykova
}

\begin{abstract}
We show that, given a compact Hausdorff space $\Omega$, there is a compact group $\mathbb{G}$ and a homeomorphic embedding of $\Omega$ into $\mathbb{G}$, such that the restriction map $\mathrm{A}(\mathbb{G}) \rightarrow C(\Omega)$ is a complete quotient map of operator spaces. In particular, this shows that there exist compact groups which contain infinite cb-Helson subsets, answering a question raised by Choi and Samei. A negative result from that paper is also improved.
\end{abstract}

\section{INTRODUCTION}

The notion of a cb-Helson subset of a locally compact group was introduced in [4], in connection with the study of quotients of Fourier algebras. For instance, the following result can be obtained by an easy modification of the proof of $[4$, Theorem B].

Theorem 1.1 (Corollary of work in [4]). Let $G$ be a SIN group, and let $J$ be a closed ideal in the Fourier algebra $\mathrm{A}(G)$. Suppose that $\mathrm{A}(G) / J$ is completely boundedly isomorphic to a closed subalgebra of $\mathcal{B}(\mathcal{H})$ for some Hilbert space $\mathcal{H}$. Then there is a cb-Helson subset $E \subset G$ such that $J=\left\{f \in \mathrm{A}(G):\left.f\right|_{E}=0\right\}$.

We defer the definition of a cb-Helson set to Section 2. For now, we note that such sets appear to be rather hard to come by: finite subsets of locally compact groups have the cb-Helson property, but hitherto no infinite cb-Helson sets were

Copyright 2016 by the Tusi Mathematical Research Group.

Received Apr. 9, 2015; Accepted Jul. 30, 2015.

2010 Mathematics Subject Classification. Primary 43A30; Secondary 46L07.

Keywords. Fourier algebra, Helson set, operator space. 


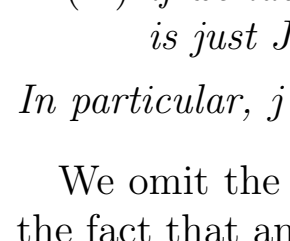

Ann. Funct. Anal. 7 (2016), no. 1, 158-169

http://dx.doi.org/10.1215/20088752-3429526

ISSN: 2008-8752 (electronic)

http://projecteuclid.org/afa

\title{
REALIZATION OF COMPACT SPACES AS CB-HELSON SETS
}

\author{
YEMON CHOI \\ Dedicated to Professor Anthony To-Ming Lau, with thanks for all his work on behalf of the \\ international community in abstract harmonic analysis \\ Communicated by Z. Lykova
}

\begin{abstract}
We show that, given a compact Hausdorff space $\Omega$, there is a compact group $\mathbb{G}$ and a homeomorphic embedding of $\Omega$ into $\mathbb{G}$, such that the restriction map $\mathrm{A}(\mathbb{G}) \rightarrow C(\Omega)$ is a complete quotient map of operator spaces. In particular, this shows that there exist compact groups which contain infinite cb-Helson subsets, answering a question raised by Choi and Samei. A negative result from that paper is also improved.
\end{abstract}

\section{INTRODUCTION}

The notion of a cb-Helson subset of a locally compact group was introduced in [4], in connection with the study of quotients of Fourier algebras. For instance, the following result can be obtained by an easy modification of the proof of $[4$, Theorem B].

Theorem 1.1 (Corollary of work in [4]). Let $G$ be a SIN group, and let $J$ be a closed ideal in the Fourier algebra $\mathrm{A}(G)$. Suppose that $\mathrm{A}(G) / J$ is completely boundedly isomorphic to a closed subalgebra of $\mathcal{B}(\mathcal{H})$ for some Hilbert space $\mathcal{H}$. Then there is a cb-Helson subset $E \subset G$ such that $J=\left\{f \in \mathrm{A}(G):\left.f\right|_{E}=0\right\}$.

We defer the definition of a cb-Helson set to Section 2. For now, we note that such sets appear to be rather hard to come by: finite subsets of locally compact groups have the cb-Helson property, but hitherto no infinite cb-Helson sets were

Copyright 2016 by the Tusi Mathematical Research Group.

Received Apr. 9, 2015; Accepted Jul. 30, 2015.

2010 Mathematics Subject Classification. Primary 43A30; Secondary 46L07.

Keywords. Fourier algebra, Helson set, operator space. 


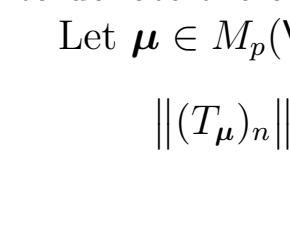

Ann. Funct. Anal. 7 (2016), no. 1, 158-169

http://dx.doi.org/10.1215/20088752-3429526

ISSN: 2008-8752 (electronic)

http://projecteuclid.org/afa

\title{
REALIZATION OF COMPACT SPACES AS CB-HELSON SETS
}

\author{
YEMON CHOI \\ Dedicated to Professor Anthony To-Ming Lau, with thanks for all his work on behalf of the \\ international community in abstract harmonic analysis \\ Communicated by Z. Lykova
}

\begin{abstract}
We show that, given a compact Hausdorff space $\Omega$, there is a compact group $\mathbb{G}$ and a homeomorphic embedding of $\Omega$ into $\mathbb{G}$, such that the restriction map $\mathrm{A}(\mathbb{G}) \rightarrow C(\Omega)$ is a complete quotient map of operator spaces. In particular, this shows that there exist compact groups which contain infinite cb-Helson subsets, answering a question raised by Choi and Samei. A negative result from that paper is also improved.
\end{abstract}

\section{INTRODUCTION}

The notion of a cb-Helson subset of a locally compact group was introduced in [4], in connection with the study of quotients of Fourier algebras. For instance, the following result can be obtained by an easy modification of the proof of $[4$, Theorem B].

Theorem 1.1 (Corollary of work in [4]). Let $G$ be a SIN group, and let $J$ be a closed ideal in the Fourier algebra $\mathrm{A}(G)$. Suppose that $\mathrm{A}(G) / J$ is completely boundedly isomorphic to a closed subalgebra of $\mathcal{B}(\mathcal{H})$ for some Hilbert space $\mathcal{H}$. Then there is a cb-Helson subset $E \subset G$ such that $J=\left\{f \in \mathrm{A}(G):\left.f\right|_{E}=0\right\}$.

We defer the definition of a cb-Helson set to Section 2. For now, we note that such sets appear to be rather hard to come by: finite subsets of locally compact groups have the cb-Helson property, but hitherto no infinite cb-Helson sets were

Copyright 2016 by the Tusi Mathematical Research Group.

Received Apr. 9, 2015; Accepted Jul. 30, 2015.

2010 Mathematics Subject Classification. Primary 43A30; Secondary 46L07.

Keywords. Fourier algebra, Helson set, operator space. 


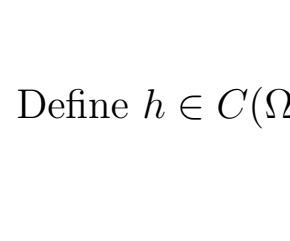

Ann. Funct. Anal. 7 (2016), no. 1, 158-169

http://dx.doi.org/10.1215/20088752-3429526

ISSN: 2008-8752 (electronic)

http://projecteuclid.org/afa

\title{
REALIZATION OF COMPACT SPACES AS CB-HELSON SETS
}

\author{
YEMON CHOI \\ Dedicated to Professor Anthony To-Ming Lau, with thanks for all his work on behalf of the \\ international community in abstract harmonic analysis \\ Communicated by Z. Lykova
}

\begin{abstract}
We show that, given a compact Hausdorff space $\Omega$, there is a compact group $\mathbb{G}$ and a homeomorphic embedding of $\Omega$ into $\mathbb{G}$, such that the restriction map $\mathrm{A}(\mathbb{G}) \rightarrow C(\Omega)$ is a complete quotient map of operator spaces. In particular, this shows that there exist compact groups which contain infinite cb-Helson subsets, answering a question raised by Choi and Samei. A negative result from that paper is also improved.
\end{abstract}

\section{INTRODUCTION}

The notion of a cb-Helson subset of a locally compact group was introduced in [4], in connection with the study of quotients of Fourier algebras. For instance, the following result can be obtained by an easy modification of the proof of $[4$, Theorem B].

Theorem 1.1 (Corollary of work in [4]). Let $G$ be a SIN group, and let $J$ be a closed ideal in the Fourier algebra $\mathrm{A}(G)$. Suppose that $\mathrm{A}(G) / J$ is completely boundedly isomorphic to a closed subalgebra of $\mathcal{B}(\mathcal{H})$ for some Hilbert space $\mathcal{H}$. Then there is a cb-Helson subset $E \subset G$ such that $J=\left\{f \in \mathrm{A}(G):\left.f\right|_{E}=0\right\}$.

We defer the definition of a cb-Helson set to Section 2. For now, we note that such sets appear to be rather hard to come by: finite subsets of locally compact groups have the cb-Helson property, but hitherto no infinite cb-Helson sets were

Copyright 2016 by the Tusi Mathematical Research Group.

Received Apr. 9, 2015; Accepted Jul. 30, 2015.

2010 Mathematics Subject Classification. Primary 43A30; Secondary 46L07.

Keywords. Fourier algebra, Helson set, operator space. 


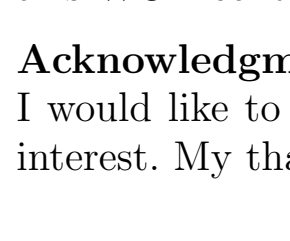

Ann. Funct. Anal. 7 (2016), no. 1, 158-169

http://dx.doi.org/10.1215/20088752-3429526

ISSN: 2008-8752 (electronic)

http://projecteuclid.org/afa

\title{
REALIZATION OF COMPACT SPACES AS CB-HELSON SETS
}

\author{
YEMON CHOI \\ Dedicated to Professor Anthony To-Ming Lau, with thanks for all his work on behalf of the \\ international community in abstract harmonic analysis \\ Communicated by Z. Lykova
}

\begin{abstract}
We show that, given a compact Hausdorff space $\Omega$, there is a compact group $\mathbb{G}$ and a homeomorphic embedding of $\Omega$ into $\mathbb{G}$, such that the restriction map $\mathrm{A}(\mathbb{G}) \rightarrow C(\Omega)$ is a complete quotient map of operator spaces. In particular, this shows that there exist compact groups which contain infinite cb-Helson subsets, answering a question raised by Choi and Samei. A negative result from that paper is also improved.
\end{abstract}

\section{INTRODUCTION}

The notion of a cb-Helson subset of a locally compact group was introduced in [4], in connection with the study of quotients of Fourier algebras. For instance, the following result can be obtained by an easy modification of the proof of $[4$, Theorem B].

Theorem 1.1 (Corollary of work in [4]). Let $G$ be a SIN group, and let $J$ be a closed ideal in the Fourier algebra $\mathrm{A}(G)$. Suppose that $\mathrm{A}(G) / J$ is completely boundedly isomorphic to a closed subalgebra of $\mathcal{B}(\mathcal{H})$ for some Hilbert space $\mathcal{H}$. Then there is a cb-Helson subset $E \subset G$ such that $J=\left\{f \in \mathrm{A}(G):\left.f\right|_{E}=0\right\}$.

We defer the definition of a cb-Helson set to Section 2. For now, we note that such sets appear to be rather hard to come by: finite subsets of locally compact groups have the cb-Helson property, but hitherto no infinite cb-Helson sets were

Copyright 2016 by the Tusi Mathematical Research Group.

Received Apr. 9, 2015; Accepted Jul. 30, 2015.

2010 Mathematics Subject Classification. Primary 43A30; Secondary 46L07.

Keywords. Fourier algebra, Helson set, operator space. 